\title{
Competition between Burkholderia pseudomallei and $B$. thailandensis
}

\author{
Wikanda Ngamdee ${ }^{1}$, Sarunporn Tandhavanant ${ }^{1,2}$, Chanthiwa Wikraiphat ${ }^{2}$, Onrapak Reamtong ${ }^{3}$, Vanaporn Wuthiekanun², \\ Jeanne Salje, , David A Low ${ }^{4,5}$, Sharon J Peacock ${ }^{1,2,6}$ and Narisara Chantratita, ${ }^{1,2 *}$
}

\begin{abstract}
Background: Burkholderia pseudomallei is a Gram-negative bacterium that causes melioidosis, an often fatal disease in tropical countries. Burkholderia thailandensis is a non-virulent but closely related species. Both species are soil saprophytes but are almost never isolated together.

Results: We identified two mechanisms by which $B$. pseudomallei affects the growth of $B$. thailandensis. First, we found that six different isolates of $B$. pseudomallei inhibited the growth of $B$. thailandensis on LB agar plates. Second, our results indicated that $55 \%$ of isolated strains of $B$. pseudomallei produced a secreted compound that inhibited the motility but not the viability of $B$. thailandensis. Analysis showed that the active compound was a $\mathrm{pH}$-sensitive and heat-labile compound, likely a protein, which may affect flagella processing or facilitate their degradation. Analysis of bacterial sequence types (STs) demonstrated an association between this and motility inhibition. The active compound was produced from B. pseudomallei during the stationary growth phase.

Conclusion: Taken together, our results indicate that B. pseudomallei inhibits both the growth and motility of its close relative $B$. thailandensis. The latter phenomenon appears to occur via a previously unreported mechanism involving flagellar processing or degradation.
\end{abstract}

Keywords: Burkholderia pseudomallei, B. thailandensis, Melioidosis, Swarming, Flagella, Competitive growth inhibition

\section{Background}

B. pseudomallei is a Gram-negative bacillus and the cause of melioidosis. Most cases of infection are reported from northeast Thailand and northern Australia, although an increasing number of cases are being reported from across Southeast Asia, and from Africa and south America [1]. B. pseudomallei is an environmental saprophyte, and infection arises following bacterial inoculation, inhalation or ingestion [2]. The range of clinical manifestations are broad, but the majority of patients present with an acute febrile illness associated with one or more of pneumonia, bacteremia, or abscess formation in the spleen, liver or elsewhere [3]. In Thailand this infection accounts for $20 \%$ of community-

\footnotetext{
* Correspondence: narisara@tropmedres.ac

1 Department of Microbiology and Immunology, Faculty of Tropical Medicine,

Mahidol University, 420/6 Rajvithi Road, Bangkok 10400, Thailand

${ }^{2}$ Mahidol-Oxford Tropical Medicine Research Unit, Faculty of Tropical

Medicine, Mahidol University, Bangkok, Thailand

Full list of author information is available at the end of the article
}

acquired septicemias [3], and the mortality rate is 12$40 \%$ despite appropriate antimicrobial therapy.

Environmental surveys to detect $B$. pseudomallei have been performed to address a range of questions, including mapping of geographic distribution, the effect of sampling depth and season on positivity of B. pseudomallei in soil and water, and the phylogeny of environmental isolates [4]. A striking observation arising from an intensive sampling survey conducted in a single plot of disused land $\left(237.5 \mathrm{~m}^{2}\right)$ in northeast Thailand was the genetic diversity of $B$. pseudomallei within and between sampling points. Genotyping of 600 primary culture plate colonies from 3 sampling points showed that each contained three or four different B. pseudomallei clones, with little overlap in genotype between samples but a predominance of a single genotype within a given sample [4]. One explanation for bacterial population structuring within a single sample is that the predominant clone has a competitive advantage over other B. pseudomallei lineages and/or other microbial species or genera. 
B. pseudomallei is genetically closely related to the non-pathogenic $B$. thailandensis [5,6], which is also present in the environment including geographic areas that are positive for B. pseudomallei. Although B. thailandensis is rarely searched for systematically during environmental surveys of $B$. pseudomallei, B. thailandensis grows on the same culture media and the colony morphology on agar can be difficult to distinguish from $B$. pseudomallei. As a result, B. thailandensis is frequently isolated during soil sampling and discarded after identification. A review of environmental study indicates that $B$. pseudomallei and B. thailandensis are rarely isolated from the same sampling location [7]. A study 232 soil isolates from 4 regions of Thailand reported that the ratio of B. pseudomallei to B. thailandensis was 1.7, 0.9, 0.5 and 0.4 in northeastern, southern, northern and central regions, respectively [7]. The higher ratio of $B$. pseudomallei and $B$. thailandensis in the northeast was associated with a higher prevalence of melioidosis in this region compared with others. One explanation for this observation is competition between B. pseudomallei and B. thailandensis.

Several studies have provided evidence for a competitive advantage of $B$. thailandensis over other environmental bacterial species through a range of mechanisms. B. thailandensis secretes an antibiotic that inhibits the growth of Bacillus subtilis [8]. Inactivation of type VI secretion systems (T6SS)-1 renders B. thailandensis more susceptible to cell contact-induced stasis by Pseudomonas putida, Pseudomonas fluorescens and Serratia proteamaculans. B. thailandensis lacking T6SS-1 is also rapidly displaced from mixed biofilms with $P$. putida, whereas wild-type persists and overgrows the competitor [9]. B. pseudomallei and B. thailandensis have also been reported to have a contact-dependent growth inhibition (CDI) system mediated by the CdiB/CdiA family of twopartner secretion proteins $[10,11]$.

The aim of this study was to use in vitro assays to investigate competition (including viability and motility) between a range of $B$. pseudomallei and B. thailandensis isolates associated with human infection (B. pseudomal$l e i$ ) and the environment (both species), and to explore the relationship between inhibition and genotype.

\section{Results}

Growth rate analysis of $B$. pseudomallei and $B$. thailandensis

Different growth rates may affect the number of viable bacteria in the growth inhibition assay. Thus, prior to observation of competition between B. pseudomallei and B. thailandensis, the individual growth of six B. pseudomallei (H1244a, 1106b, 1026b, B4, 1710a and K96243) and B. thailandensis Bt6 were compared in LB broth. Using a starting inoculum of $1 \times 10^{5} \mathrm{cfu} / \mathrm{ml}, \log$ and stationary phase occurred at $2 \mathrm{~h}$ and $12 \mathrm{~h}$, respectively, for all isolates. There was no difference in doubling time between $B$. pseudomallei and B. thailandensis. The average doubling time for B. pseudomallei K96243, H1244a, 1106b, 1026b, 1710a and B4 were 38.7, 38.3, 38.4, 38.4, 38.6 and 38.5, and for B. thailandensis Bt6 was $39.8 \mathrm{~min}$, respectively.

\section{B. pseudomallei inhibits growth of $B$. thailandensis}

Using the growth inhibition assay, we found that $B$. pseudomallei 1710a inhibited B. thailandensis Bt6 when mixed at ratios from 1000:1 to 10:1 (Figure 1A). At $24 \mathrm{~h}$ of incubation, the number of viable $B$. pseudomallei increased by 1-1.5 logs and the number of $B$. thailandensis decreased by 1.5-2.0 logs. There was no inhibition at a ratio of $1: 1$. The reduction in number of $B$. thailandensis Bt6 after co-culture with B. pseudomallei 1710a was maximal at a ratio of 1000:1.

Next, we examined inhibition at different incubation time points using a fixed ratio of $B$. pseudomallei 1710a and B. thailandensis Bt6 of 1000:1. The number of viable bacteria for B. pseudomallei and B. thailandensis at $3 \mathrm{~h}$, $6 \mathrm{~h}$ and $24 \mathrm{~h}$ following co-culture was inversely related, with levels of B. pseudomallei increasing and B. thailandensis decreasing over time (Figure 1B). Maximum inhibition was observed at $24 \mathrm{~h}$ of incubation. There was no increased inhibition or increased growth of Bt6 after $48 \mathrm{~h}$ (data not shown).

We examined whether other B. pseudomallei isolates could inhibit growth of $B$. thailandensis Bt6. The coculture was repeated for each of five clinical B. pseudomallei isolates (K96243, H1244a, 1106b, 1026b, 1710a) and one soil B. pseudomallei isolate (B4) against B. thailandensis Bt6 at a ratio of 1000:1. This demonstrated a 1 to $2 \log$ increase for six different B. pseudomallei isolates and a 1 to $3 \log$ decrease in B. thailandensis Bt6 (Figure 1C). The highest level of inhibition was observed with the pair B. pseudomallei 1710a and $B$. thailandensis Bt6. These data suggest that multiple $B$. pseudomallei isolates are able to inhibit the growth of B. thailandensis.

B. pseudomallei inhibits $B$. thailandensis swarming motility via a growth-independent inhibition mechanism

The second part of our study was initiated by the observation that in the growth inhibition assay described above, a colony of $B$. thailandensis Bt 6 on LB agar failed to grow to the edge of an adjacent colony of B. pseudomallei 1710a following co-culture. Using a swarm plate assay, sixty-seven $B$. pseudomallei isolates were tested against each of five $B$. thailandensis isolates, in which $B$. pseudomallei and B. thailandensis were spotted on different poles of an agar plate (Methods, method (i)). We observed that $B$. thailandensis swarmed more rapidly 


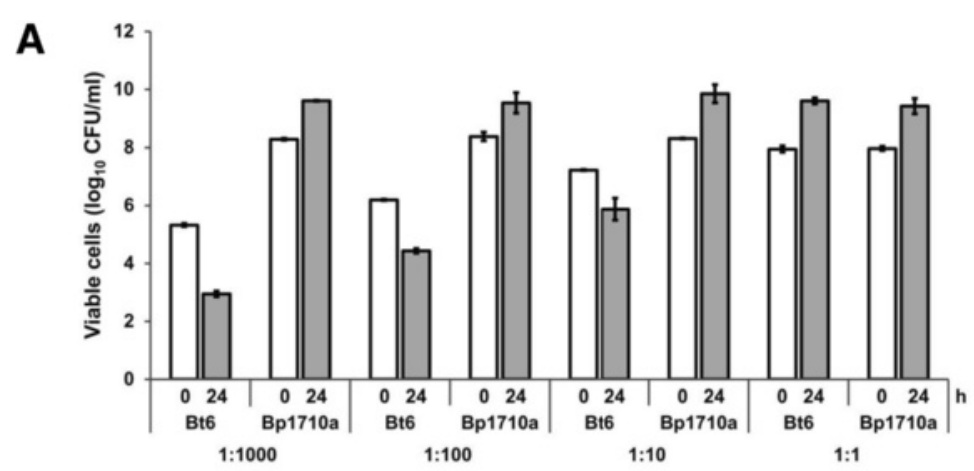

B
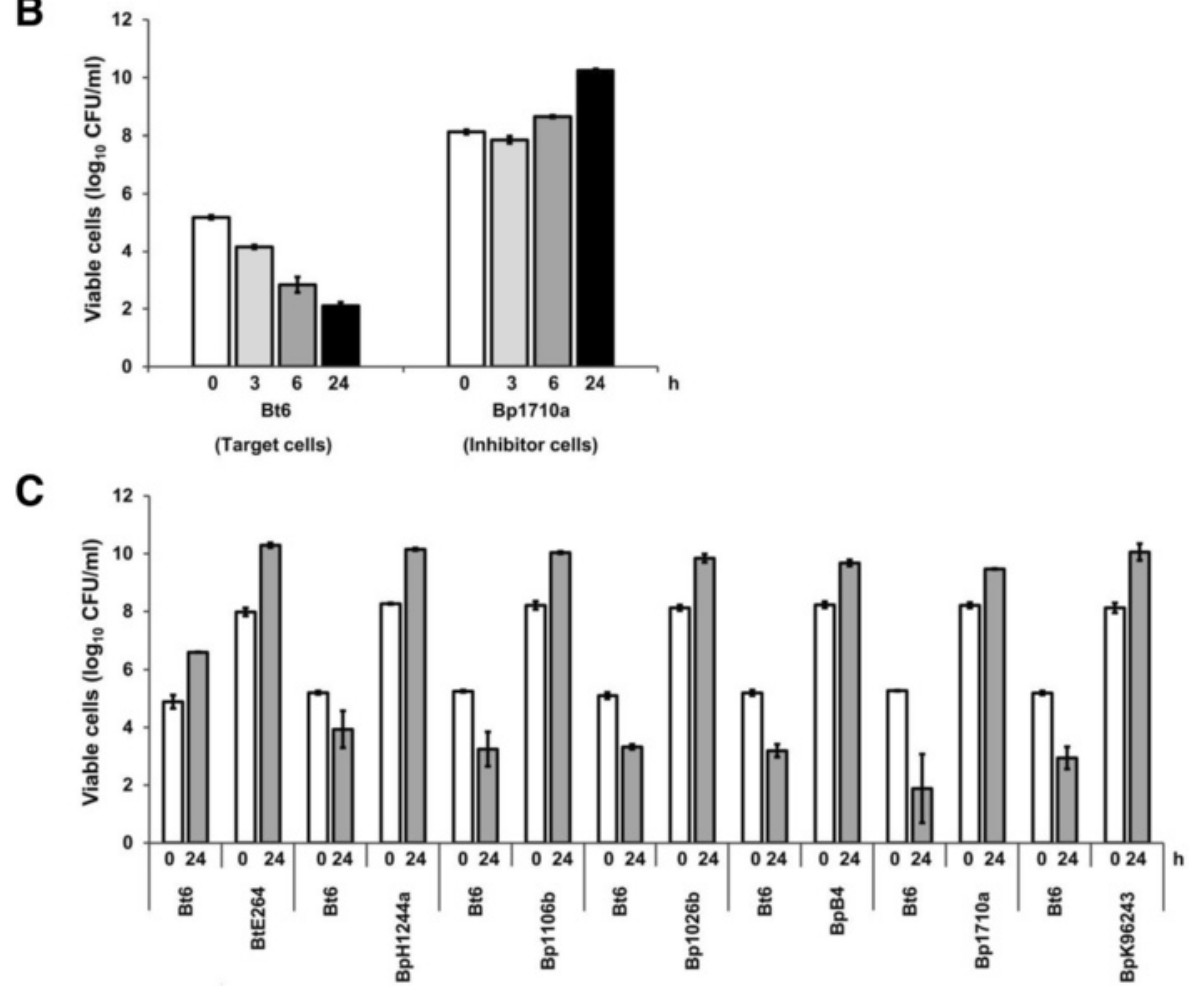

Figure 1 Growth inhibition of $B$. thailandensis Bt6 by $B$. pseudomallei 1710a on LB agar at $37^{\circ} \mathrm{C}$. (A) B. thailandensis Bt6 (target) and $B$. pseudomallei 1710a (inhibitor) cells were mixed at different inhibitor to target cell ratios and viable counts were measured at $0 \mathrm{~h}$ and $24 \mathrm{~h}$. (B) Time Course. Viable cell counts were determined at the indicated times using an inhibitor to target ratio of 1000:1 (C) Growth competition analysis of B. thailandensis Bt6 with six B. pseudomallei isolates was carried out at inhibitor to target cell ratios of 1000:1 at $0 \mathrm{~h}$ and $24 \mathrm{~h}$. A control growth competition was carried out between B. thailandensis Bt6 and B. thailandensis E264.

than B. pseudomallei, and classified the interaction between the two species into two types as follows: (1) evidence of inhibition, with a complete or partial zone of inhibition around the B. pseudomallei colony and mounding of $B$. thailandensis growth surrounding this zone (e.g. strain 1710a, Figure 2A); or (2) no evidence of inhibition, with $B$. thailandensis swarming over the entire B. pseudomallei colony (e.g. strain K96243, Figure 2A). Thirty-seven out of sixty-seven $B$. pseudomallei showed evidence of inhibitory activity against $B$. thailandensis. A consistent result was observed for a given $B$. pseudomallei isolate against each of the five $B$. thailandensis isolates.
We next explored whether the inhibition of colony migration observed was due to a secreted factor (method ii). We tested the cell-free supernatants of sixty-seven $B$. pseudomallei isolates for the inhibition of swarming of five $B$. thailandensis isolates. Inhibition was evident for the same thirty-seven B. pseudomallei isolates (55.2\%) defined by method (i), and as before each B. pseudomallei isolate gave a consistent inhibition result to each of the five $B$. thailandensis isolates (Additional file 1: Table $\mathrm{S} 1)$. When inhibition occurred, the zone containing cellfree $B$. pseudomallei supernatant was clear and was surrounded by piled up $B$. thailandensis (Figure 2B). 


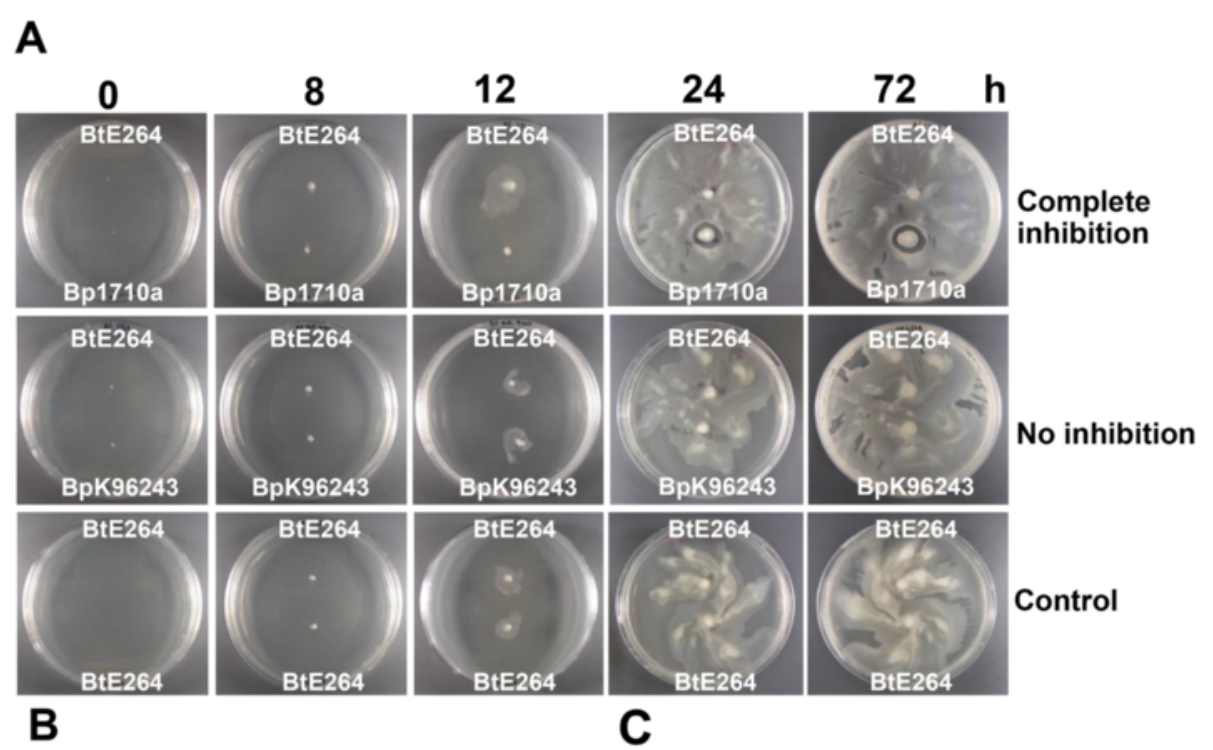

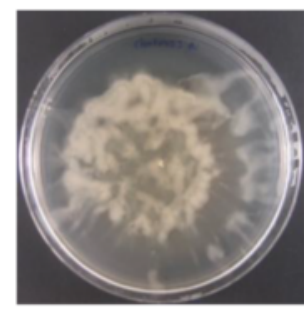

BtE264 Control

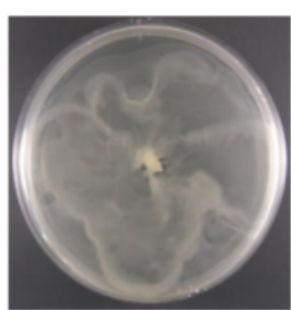

BtE264 treated by

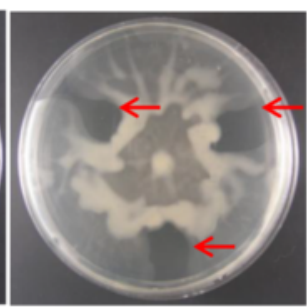

BtE264 treated by Bp1710a supernatant

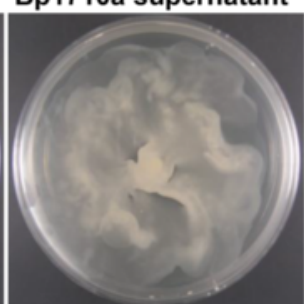

BtE264 treated by

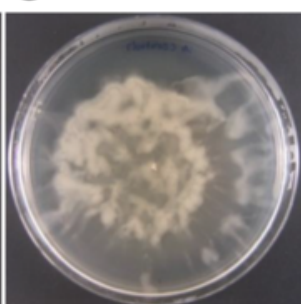

BtE264 Control

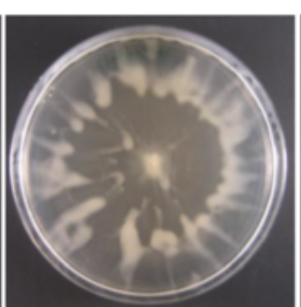

BtE264 treated with

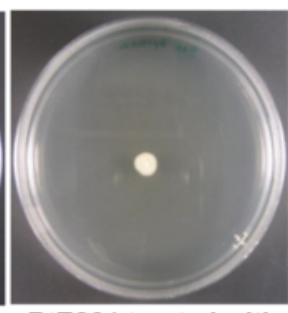

BtE264 treated with Bp1710a supernatant

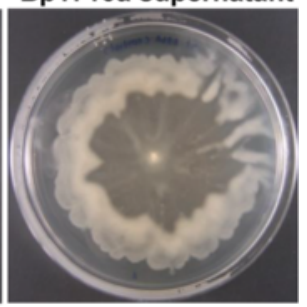

BtE264 treated with BpK96243 supernatant BtE264 supernatant BpK96243 supernatant BtE264 supernatant

Figure 2 Interaction between B. pseudomallei and B. thailandensis pairs on swarm agar. Three methods were used to detect interactions between the two species. (A) B. pseudomallei and B. thailandensis were spotted on different poles of a swarm agar plate and the growth observed daily over a $72 \mathrm{~h}$ incubation time course. An example of "Complete inhibition" is shown in which a clear zone was present around the Bp1710a strain. In contrast, no clear zone was observed around BpK96243 ("No inhibition") or control strain B. thailandensis E264. (B) Inhibitory effect of B. pseudomallei cell-free supernatant on B. thailandensis E264 motility on swarm agar. A B. thailandensis colony was spotted onto the center of swarm agar and incubated at $37^{\circ} \mathrm{C}$ for $16 \mathrm{~h}$ to allow initial swarming to begin, after which three drops of B. pseudomallei cell-free supernatant were inoculated at 2 , 6 and 10 o'clock followed by further incubation for 16 hours. Swarming of B. thailandensis E264 was blocked around three spots of supernatant from $B$. pseudomallei 1710a supernatant (arrows), and is representative of results using strains that inhibited B. thailandensis swarming. B. pseudomallei K96243 is representative of strains that did not inhibit $B$. thailandensis swarming. Controls: $B$. thailandensis E264 colony with no supernatant added (top left panel), and B. thailandensis E264 colony with B. thailandensis E264 supernatant added (bottom right panel). (C) The inhibitory effect of B. pseudomallei cell-free supernatant on B. thailandensis E264 colony swarming. One hundred microliters of cell-free supernatant from B. pseudomallei was deposited at the center of a swarm agar plate and left to dry in air for $15 \mathrm{~min}$. Subsequently, B. thailandensis were spotted at the center of the plate. Growth of the swarm colony was observed after incubation at $37^{\circ} \mathrm{C}$ for $18 \mathrm{~h}$.

A further experiment was performed to explore these findings using the same sixty-seven $B$. pseudomalle $i$ isolates. When cell-free supernatant of $B$. pseudomallei was spotted onto swarm agar, left to dry for 15 min and then overlaid by $B$. thailandensis cells (method iii), we observed two outcomes; $B$. thailandensis failed to swarm when overlaid on supernatant from the same thirtyseven $B$. pseudomallei, while in the remainder B. thailandensis swarmed without evidence of inhibition (Figure 2C). Again, each B. pseudomallei showed the 
same results for five different $B$. thailandensis isolates. Based on these results, we divided B. pseudomallei into two groups based on the presence or absence of swarm inhibitory activity.

\section{Cell-free supernatant of $B$. pseudomallei does not inhibit growth of $B$. thailandensis}

Since the supernatant of B. pseudomallei 1710a inhibited $B$. thailandensis E264 swarming motility, we sought to investigate whether the supernatant of $B$. pseudomallei affected viability of $B$. thailandensis. A broth microdilution assay was used to measure growth inhibition. We found that the number of viable $B$. thailandensis cells did not decrease during incubation at $37^{\circ} \mathrm{C}$ during exposure to different concentrations of cell-free supernatant from B. pseudomallei 1710a, B. pseudomallei K96243 and B. thailandensis E264 control, and the number of treated B. thailandensis E264 cells counts were comparable to that of the untreated control (data not shown). This result suggests that the secreted factor (denoted here as "inhibitory factor") does not function as growth inhibitor but only inhibited $B$. thailandensis swarming.

\section{Inhibitory activity is associated with genotype}

The B. pseudomallei multilocus sequence type (ST) was known for sixty-six out of sixty-seven isolates. The frequency of inhibition was defined for each ST, which demonstrated an association between the two groups. Inhibition was observed in 14 STs represented by 36 isolates, but not in $10 \mathrm{STs}$ represented by 30 isolates. However, $5 \mathrm{STs}$ contained both inhibitory and non-inhibitory isolates (Table 1). This included $12 / 13$ of ST54 that were able to inhibit B. thailandensis, and $18 / 20$ of ST70 lacking swarm inhibitory activity $(\mathrm{P}<0.001)$.

\section{Inhibitory B. pseudomallei strains were isolated from different sources}

By comparing the effect on B. thailandensis motility on swarm agar, we did not find any significant difference between clinical and environmental isolates. Inhibition was found in 20 of 39 clinical (51.3\%) and 17 of 28 environmental isolates $(60.7 \%)(\mathrm{p}=0.44)$. The geographical origin of $B$. pseudomallei demonstrating inhibition included Thailand (36/63, 57.1\%) and Australia (1/4, 25.0\%) $(\mathrm{p}=0.32)$.

Swarm inhibitory activity is found in dominant and minor populations of $B$. pseudomallei in the same soil samples We previously demonstrated genetic diversity of $B$. pseudomallei in a single soil sample, with a predominant genotype co-existing with two or three other genotypes [4]. We hypothesized that this structuring may be
Table 1 Sequence type (ST) of 67 B. pseudomallei isolates and their corresponding inhibitory effect on $B$. thailandensis motility

\begin{tabular}{|c|c|c|c|}
\hline ST & $\begin{array}{l}\text { Total } \\
\text { tested }\end{array}$ & $\begin{array}{l}\text { Inhibition present* } \\
(n=37)\end{array}$ & $\begin{array}{l}\text { Inhibition absent* } \\
(\mathrm{n}=30)\end{array}$ \\
\hline 10 & 1 & 0 & 1 \\
\hline 15 & 1 & 1 & 0 \\
\hline 33 & 1 & 1 & 0 \\
\hline 54 & 13 & 12 & 1 \\
\hline 60 & 9 & 6 & 3 \\
\hline 70 & 20 & 2 & 18 \\
\hline 93 & 1 & 1 & 0 \\
\hline 102 & 1 & 1 & 0 \\
\hline 126 & 2 & 0 & 2 \\
\hline 129 & 1 & 0 & 1 \\
\hline 132 & 1 & 1 & 0 \\
\hline 163 & 1 & 1 & 0 \\
\hline 176 & 2 & 1 & 1 \\
\hline 177 & 7 & 6 & 1 \\
\hline 185 & 1 & 1 & 0 \\
\hline 211 & 1 & 1 & 0 \\
\hline 304 & 1 & 0 & 1 \\
\hline 424 & 1 & 0 & 1 \\
\hline 501 & 1 & 1 & 0 \\
\hline $\begin{array}{l}\text { Unknown } \\
\text { (strain 164) }\end{array}$ & 1 & 1 & 0 \\
\hline Total ST & & 14 STs & $10 \mathrm{STs}$ \\
\hline
\end{tabular}

*Inhibition effect of $B$. pseudomallei cell-free supernatant on five $B$. thailandensis isolates which showed the same result for each $B$. pseudomallei isolate. The inhibition of $B$. thailandensis swarming was performed by all three types of assays which gave the same results.

influenced by the ability of some strains to secrete an inhibitory factor. We examined the inhibition of B. thailandensis motility by cell-free B. pseudomallei supernatant for each of the STs identified from 3 independent soil samples [4] (Table 2). Only two isolates failed to demonstrated swarm inhibition, one from each of two soil samples. This evidence does not support our hypothesis, although there may be other effects that would not be detected by this assay.

\section{No inhibition between pairwise testing of different $B$.} pseudomallei isolates

We further investigated the possibility that a B. pseudomallei predominant genotype in a single soil sample may use the inhibition activity to limit the other minor genotypes of the same species. Pairwise testing was examined of the inhibition of B. pseudomallei motility by cell-free B. pseudomallei supernatant for each of the STs identified from 3 independent soil samples [4]. The $B$. pseudomallei isolates analyzed are shown in Table 2. We 
Table 2 Inhibition of $B$. thailandensis motility by cell-free supernatant of $B$. pseudomallei from three independent soil samples

\begin{tabular}{|c|c|c|c|c|}
\hline Soil sample ${ }^{a}$ & Bp isolates & Sequence type & No. of colonies ${ }^{b}$ & Inhibition $^{c}$ \\
\hline \multirow[t]{4}{*}{ E4 } & A1 & ST424 & $38(19 \%)$ & - \\
\hline & A2 & ST177 & $12(6 \%)$ & + \\
\hline & A3 & ST176 & $10(5 \%)$ & + \\
\hline & A4 & ST185 & 140 (70\%) & + \\
\hline \multirow[t]{4}{*}{ D10 } & B1 & ST33 & $50(25 \%)$ & + \\
\hline & B2 & ST60 & $18(9 \%)$ & + \\
\hline & B3 & ST163 & 103 (51.5\%) & + \\
\hline & B4 & ST176 & $29(14.5 \%)$ & + \\
\hline \multirow[t]{3}{*}{ A11 } & $\mathrm{C} 1$ & ST93 & 174 (87\%) & + \\
\hline & C2 & ST304 & 17 (8.5\%) & - \\
\hline & C4 & ST60 & $9(4.5 \%)$ & + \\
\hline
\end{tabular}

, soil sample and culture data was obtained from our previous study [4]. The study reported the distribution of $B$. pseudomallei within an area of disused land in northeast Thailand and genotypes of primary plate colonies isolated from three independent sampling points (E4, D10 and A11).

b , Out of a total of 200 colonies per sample.

c, Inhibition effect of $B$. pseudomallei cell-free supernatant on five $B$. thailandensis isolates. The inhibition of $B$. thailandensis swarming was performed by the method (ii) assay. The table shows results of five $B$. thailandensis isolates which had the same results.

found that the culture supernatant of all B. pseudomallei isolated from soil did not inhibit motility of any other $B$. pseudomallei. We also tested the supernatant of a clinical isolate (B. pseudomallei 1710a) against itself and 66 B. pseudomallei other isolates (listed in Additional file 1: Table S1) and no inhibition were observed. This suggests that the role of this secreted factor may be to inhibit the motility of other species.

Three isogenic morphotypes showed the same inhibition activity results

We previously reported that $B$. pseudomallei can switch colony morphology to different types under stress conditions $[12,13]$. We next investigated whether the secretion of inhibitory factor in a given isolate is related to colony morphotype. Three isogenic morphotypes referred as types I, II and III generated from wild-type type I for each five B. pseudomallei strains (153, 164, K96243, B3 and B4) were tested against each of five B. thailandensis strains (E29, E175, E264, E421 and E426) using the inhibition assay method (ii). B. pseudomallei strains 153, 164 and K96243 were clinical isolates and B3 and B4 were soil isolates. All three colony morphotypes of strains 153, 164 and B4 showed inhibition, whilst all three types of K96243 and B3 did not. This result indicated that the secretion of inhibitory factor by B. pseudomallei is strain-dependent but not related to the morphotypes tested.

\section{B. pseudomallei secretes an inhibitory factor during stationary phase}

To determine whether the secretion of the B. pseudomallei inhibitory factor was growth-phase dependent, we cultured $1 \times 10^{5} \mathrm{cfu} / \mathrm{ml}$ B. pseudomallei 1710a in $10 \mathrm{ml} \mathrm{LB}$ broth at $37^{\circ} \mathrm{C}$ with shaking at $200 \mathrm{rpm}$ for $24 \mathrm{~h}$. The supernatant was collected every $2 \mathrm{~h}$ and filter-sterilized, and these samples examined for the presence of inhibition of B. thailandensis E264 swarming using inhibition assay method (ii). Inhibitory activity was detected in all aliquots collected after $12 \mathrm{~h}$ incubation when the $B$. pseudomallei count reached $5 \times 10^{9} \mathrm{cfu} / \mathrm{ml}$ and was in stationary phase (Figure 3). This suggests that inhibitory factor secreted by $B$. pseudomallei may be concentration dependent or bacterial density-dependent.

\section{Estimation of the mass of the inhibitory factor of $B$. pseudomallei}

Filtration through membranes with different pore sizes (10 kDa to $100 \mathrm{kDa}$ size cut-off) was used to estimate the molecular weight of the inhibitory factor from $B$. pseudomallei. Two B. pseudomallei isolates with inhibition activity (1710a and 1026b) were tested. Following filtration, 15 times concentrated volume of original supernatants were obtained and $100 \mu \mathrm{l}$ filtrate and retentate fractions were tested for the inhibition of B. thailandensis swarming using assay method (ii). The inhibition of B. thailandensis E264 swarming was detected in retentate samples from all membranes. The inhibition was not detected in the filtrate of both B. pseudomallei supernatants when $10 \mathrm{kDa}$ and $30 \mathrm{kDa}$ membranes were used, but was present in filtrate fractions from $50 \mathrm{kDa}$ and $100 \mathrm{kDa}$ membranes (Figure 4A). This result suggests that the inhibitory factor has an estimated MW of 30 to $50 \mathrm{kDa}$. 
A

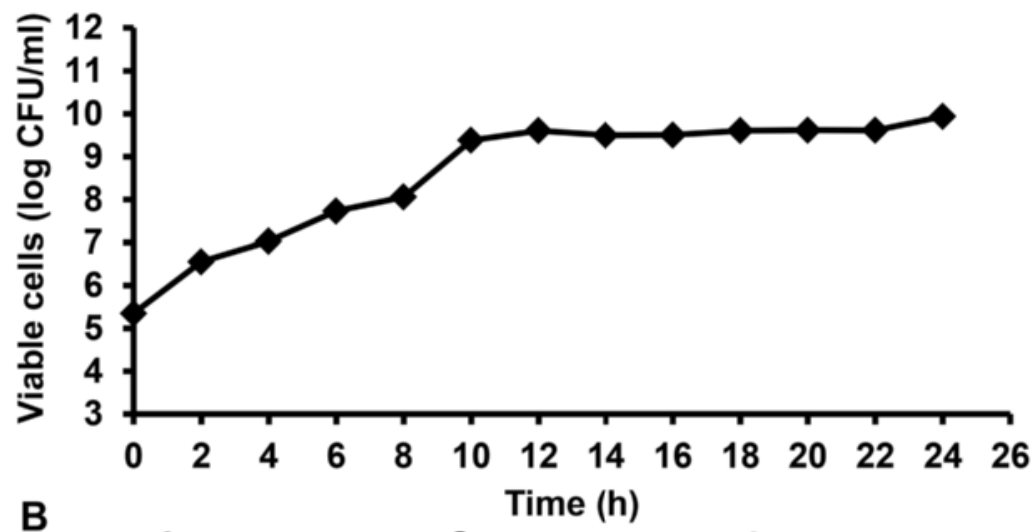

B

\section{4}

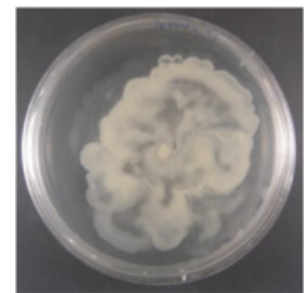

8

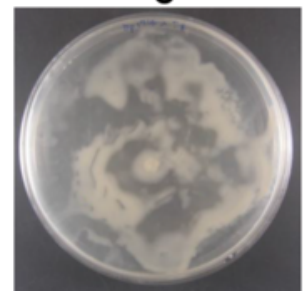

16

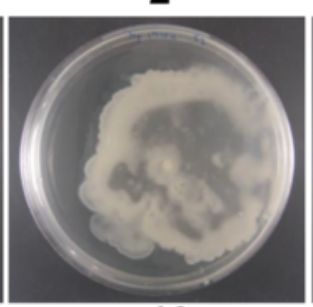

10

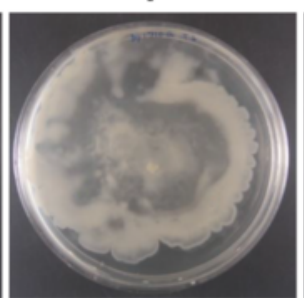

12
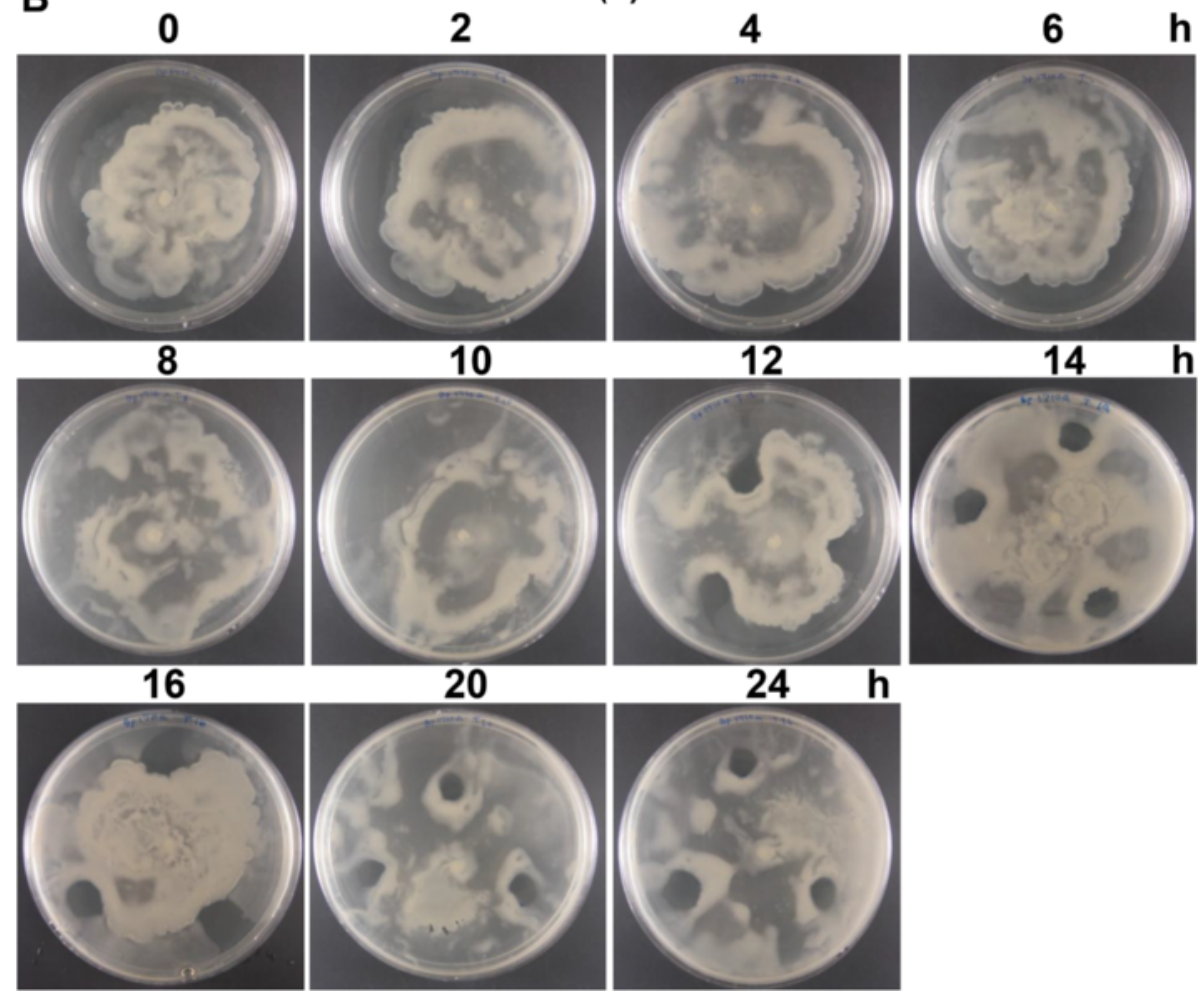

20
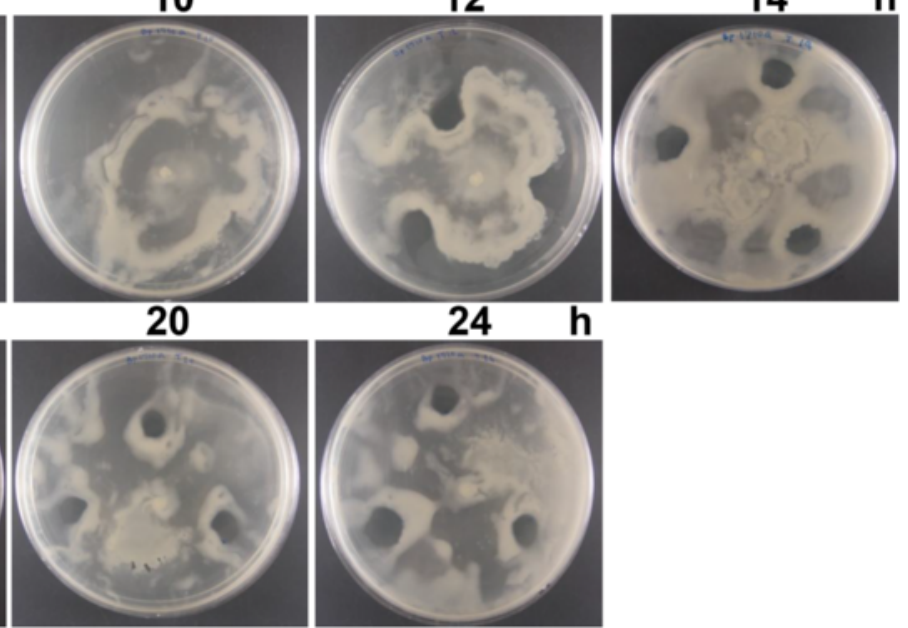

Figure 3 Inhibitory activity of cell-free supernatant of B. pseudomallei 1710a produced during growth in LB broth. (A) Growth curve of B. pseudomallei 1710a, (B) Inhibition activity of cell-free supernatant from B. pseudomallei 1710a which was collected at $2 \mathrm{~h}$ time interval during culture, with $B$. thailandensis E264 motility on swarm agar.

Effect of protein digestion on inhibitory activity of $B$. pseudomallei

Next, we determined whether the inhibitory activity was mediated by a protein or non-protein factor. We tested the $15 \mathrm{x}$-concentrated cell-free supernatant of B. pseudomallei 1710a on B. thailandensis E264 swarming, comparing the activity before and after protein digestion with each of two protease enzymes. The results shown in Figure $4 \mathrm{~B}$ demonstrated that inhibitory activity was sensitive to proteinase $\mathrm{K}$ and pronase digestion. SDSPAGE analysis demonstrated that proteinase $\mathrm{K}$ and pronase completely digested $1 \mathrm{mg}$ BSA control. Thus, the inhibitory factor appears to be a protein.

Stability of the inhibitory factor of B. pseudomallei under different conditions

We examined the effect of varying $\mathrm{pH}$, temperature and salt concentration. Inhibitory activity of culture supernatant of B. pseudomallei 1710a against swarming of $B$. thailandensis E264 was retained in samples exposed to $\mathrm{pH}$ 4, 5, 6 and 7 but disappeared at $\mathrm{pH} 3$ and 8, suggesting that the factor was inactivated at extreme $\mathrm{pH}$ 

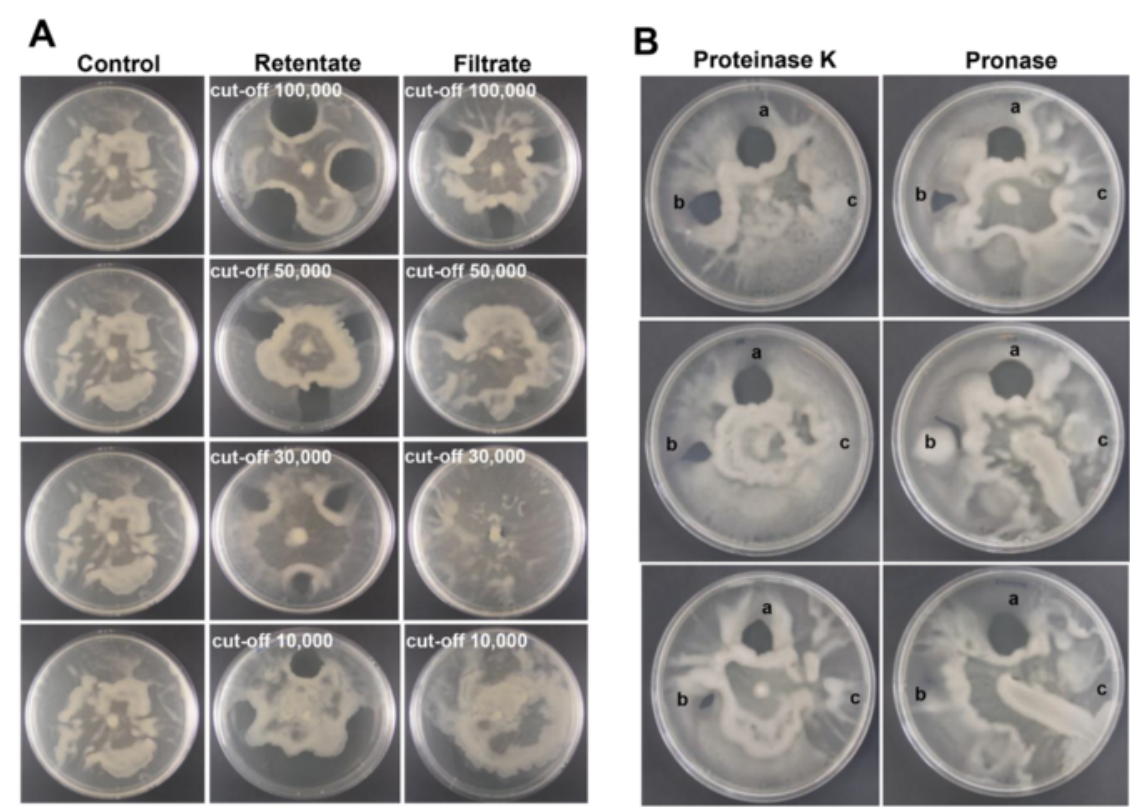

$24 h$

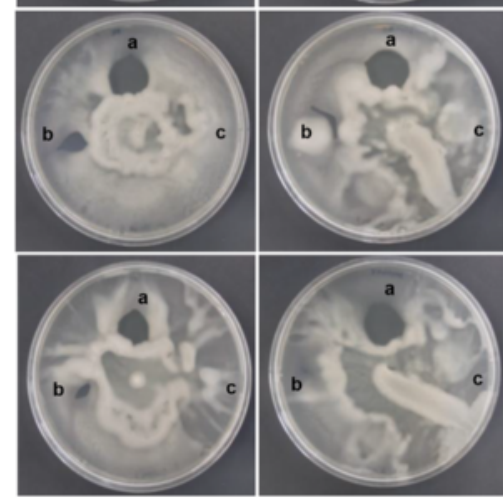

$48 \mathrm{~h}$

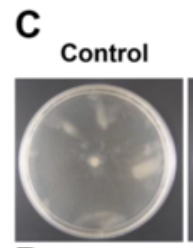

$\mathrm{pH} 3$

$\mathrm{pH} 4$
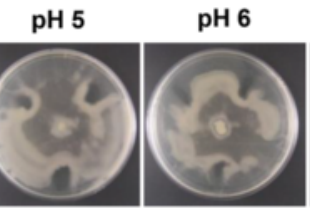

$\mathrm{pH} 7$

$\mathrm{pH} 8$
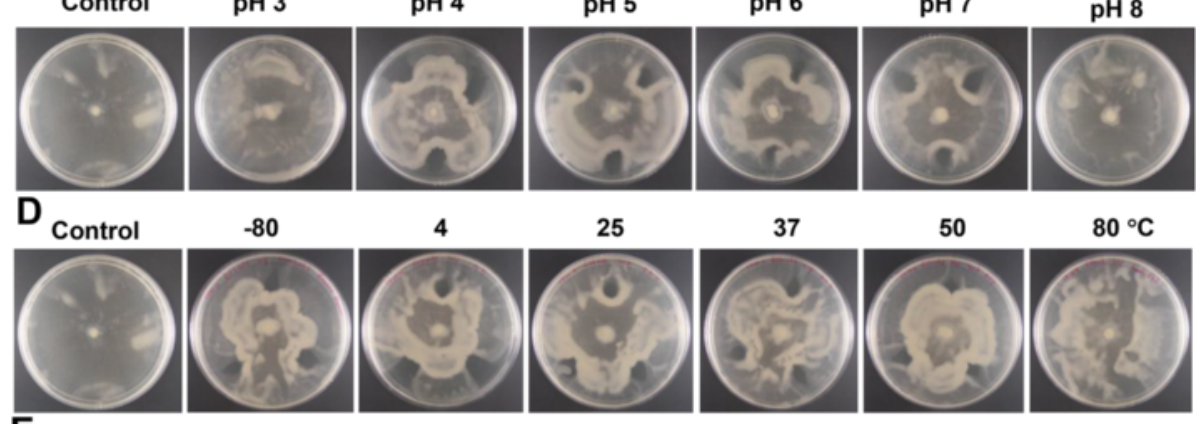

4

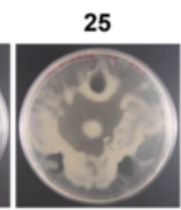

37
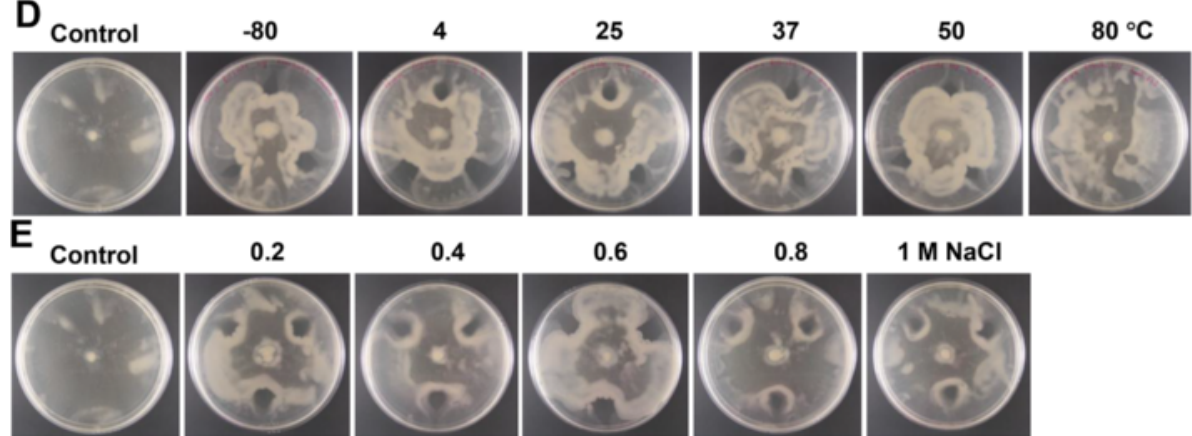

0.4

0.6

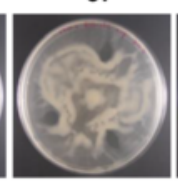

0.8

$1 \mathrm{M} \mathrm{NaCl}$
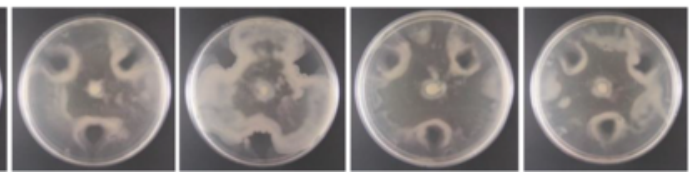

Figure 4 Inhibitory activities on B. thailandensis E264 motility on swarm agar of cell-free supernatant of B. pseudomallei 1710a after filtration, protein digestion, or incubation under different conditions. (A) Inhibition zone in retentate and filtrate fractions following filtration through membranes with different molecular weight cut-off sizes. (B) Inhibitory activity of cell-free supernatant of B. pseudomallei 1710a after treatments with proteinase K (left panel) and pronase (right panel), (a) no treatment control, (b) treatment with proteinase $\mathrm{K}$ or pronase, (c) Enzymes alone (no supernatants) were spotted onto the agar in the same concentration as a control. (C) Inhibition activity of cell-free supernatant of B. pseudomallei 1710a following incubation at different $\mathrm{pH}$ at $37^{\circ} \mathrm{C}$ for $24 \mathrm{~h}$. (D) Inhibition activity of cell-free supernatant of $B$. pseudomallei 1710a following incubation at different temperatures for $24 \mathrm{~h}$. (E) Inhibition activity of cell-free supernatant of $B$. pseudomallei 1710a following incubation at different salt concentrations at $37^{\circ} \mathrm{C}$ for $24 \mathrm{~h}$. The controls for (A), (C), (D) and (E) are B. thailandensis E264 swarming without the inoculation of cell-free supernatant. Plates were incubated at $37^{\circ} \mathrm{C}$ for $24 \mathrm{~h}$.

(Figure 4C). The effect of heat on inhibitory activity was tested by incubating the $B$. pseudomallei culture supernatant at $\mathrm{pH} 7.0$ at different temperatures for $24 \mathrm{~h}$ followed by testing in the inhibitory plate swam assay using method (ii). Incubation of supernatant at $-80^{\circ} \mathrm{C}, 4^{\circ} \mathrm{C}, 25^{\circ} \mathrm{C}, 37^{\circ} \mathrm{C}$ and $50^{\circ} \mathrm{C}$ had no effect while the activity was lost when the sample was incubated at $80^{\circ} \mathrm{C}$. The data indicates that the inhibition activity is heat-labile (Figure 4D). Variable salt concentrations achieved by adding $\mathrm{NaCl}$ to $0.2 \mathrm{M}, 0.4 \mathrm{M}, 0.6 \mathrm{M}, 0.8 \mathrm{M}$ and $1 \mathrm{M}$ to the $B$. pseudomallei $1710 \mathrm{a}$ supernatant at pH 7.0 did not affect the inhibitory activity (Figure 4E).

\section{Inhibition is associated with motility defects but not flagella expression by $B$. thailandensis}

Under light microscopy and video capture, we observed that B. thailandensis E264 exposed to supernatant of $B$. 
pseudomallei 1710a (inhibitory strain) had reduced motility compared to that after exposure to supernatant from B. pseudomallei K96243 (non-inhibitory strain), B. thailandensis E264, or the unexposed control (Figure 5A-D). Measurement of motility showed a reduction in the average distance moved by $B$. thailandensis E264 cells exposed to supernatant of B. pseudomallei 1710a compared with the non-exposed control or exposure to supernatant from $B$. pseudomallei K96243 or B. thailandensis E264 (Figure 5E).

The effect on motility was further examined used transmission electron microscopy (TEM) to determine the proportion of flagellated bacteria. Following exposure to cell-free supernatant of B. pseudomallei 1710a, the proportion of flagellated B. thailandensis E264 cells was $43 \%$ (43/100), compared with $77 \%, 81 \%$ and $95 \%$ of $B$. thailandensis E264 cells exposed to supernatant of $B$. pseudomallei K96243, B. thailandensis E264 and nonexposed control, respectively (Figure 6). The flagella of B. thailandensis E264 cells exposed to B. pseudomallei 1710a supernatant appeared to be truncated, damaged and fragmented (Figure 6). In contrast, intact flagella were observed for $B$. thailandensis E264 cells exposed to supernatant of B. pseudomallei K96243 and the nonexposed control.

RT-PCR experiments was performed to measure the expression of the $\mathrm{fliC}$ gene in B. thailandensis E264 exposed to cell-free supernatant of $B$. pseudomallei 1710a, B. pseudomallei K96243, B. thailandensis E264 and non-exposed control. No difference in gene expression was observed (Figure 5F). These results suggest that the inhibition of B. thailandensis E264 motility is not due to reduction of $f l i C$ transcription but appears to be caused by flagellar processing or damage.

\section{Discussion}

The observation that B. pseudomallei and B. thailandensis are rarely isolated from the same soil samples led us to examine whether these bacterial species compete in a range of in vitro model systems. We found that $B$. pseudomallei isolates inhibit growth of $B$. thailandensis. We demonstrated that clinical and environmental B. pseudomallei isolates with at least four different CDI types [10] suppressed $B$. thailandensis growth on LB agar plates. Notably, our results also demonstrated that $55 \%$ of $B$. pseudomallei tested secreted a protein, "inhibitory factor" that hindered B. thailandensis motility through flagellar processing such as assembly or secretion, or flagellar degradation. The inhibitory factor of B. pseudomallei is a heat-labile protein which functioned over a limited $\mathrm{pH}$ range. Our attempts to purify and identify the inhibitory factor from four liters of $B$. pseudomallei culture using gel-filtration and ion-exchange chromatography failed, which may have been due to low abundance and/or instability of the factor. Further studies using alternative approaches such as identification of the gene(s) coding for inhibitory factor and overexpression of the factor will be necessary for further characterization.

Studies in Lao PDR and Australia have revealed that soil collected in different seasons contain different numbers of B. pseudomallei $[14,15]$. Environmental dissemination of $B$. pseudomallei during the rainy season may involve motility $[12,16]$. Flagella are known to be the major cell-associated factor for bacterial motility, and $B$. pseudomallei mutants defective in the structural flagellin protein (FliC), do not swarm on agar [17]. Here, we found that swarming motility of $B$. thailandensis was inhibited by half of our B. pseudomallei isolates. Our microscopy results and RT-PCR data suggested that the inhibitory secreted product did not down-regulate flagellar expression, but led to structural changes. The mechanism is unknown but could involve several potential processes such as assembly, secretion, proteolytic degradation and depolymerization.

Analysis of genotype showed that the flagellar inhibitory factor was present in all of the isolates tested from 14 STs, and was absent in all isolates from 10 other STs, although 5 STs were present in both groups. Our test isolates included the two most abundant STs in Thailand (ST70 and ST54), which reside in different clonal complexes [18], and exhibited different inhibition results. Thus, the ability to secret the inhibitory compound was related to $B$. pseudomallei genotype. In contrast, we did not find a difference in inhibitory effect on $B$. thailandensis swarming motility for three isogenic morphotypes from each of five B. pseudomallei isolates, even though a previous study demonstrated that colony morphology variation represents several phenotypic differences [12].

One explanation for B. pseudomallei population structuring within a single soil sample is that the predominant clone may have a greater ability to inhibit $B$. thailandensis and minor populations of B. pseudomallei. We demonstrated that all B. pseudomallei isolates with the highest genotype frequency for each of three soil samples consistently inhibited motility of $B$. thailandensis. However, some minor populations also had this ability. The finding that the culture supernatant of all $B$. pseudomallei did not inhibit motility of any other $B$. pseudomallei suggests that the role of this secreted factor may be to inhibit the motility of other species. Flagellar filament of bacteria is composed of a flagellin subunit, which is diverse among bacterial species [19]. Bioinformatic analysis has demonstrated that the amino acid sequence of flagellin is conserved within each of species of B. pseudomallei and B. thailandensis. However, sequence alignment between several isolates of the two species has revealed a consistent 5 amino acid deletion at positions 247-251 (SPSFQ) in the flagellin of $B$. 

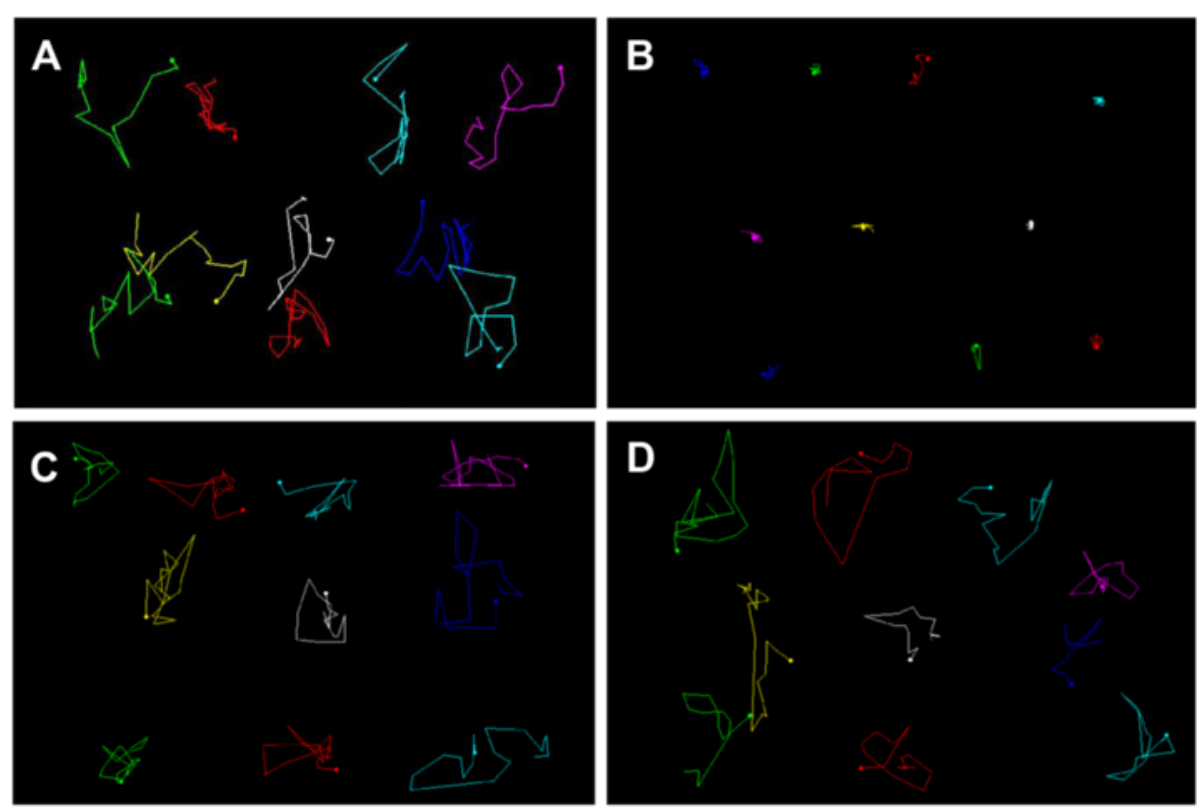

E

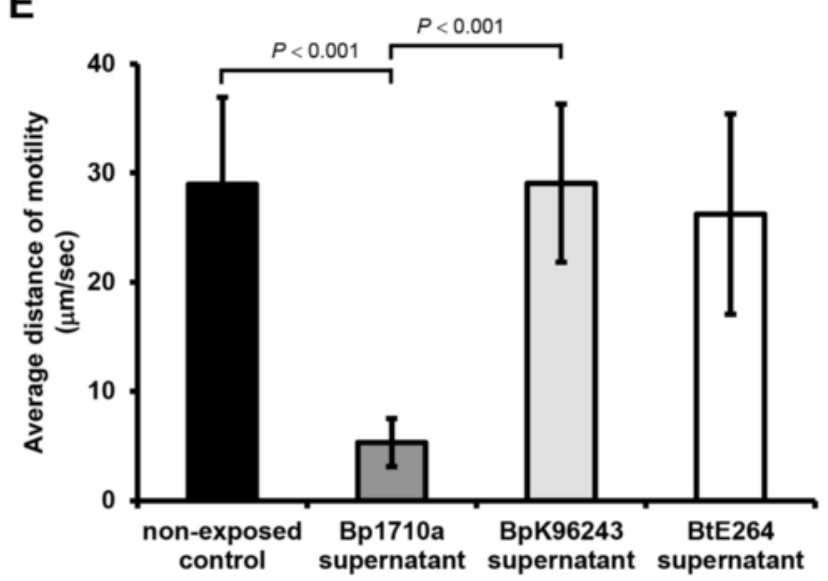

\section{Enon-exposed control \\ 口Bp1710a supernatant \\ 口BpK96243 supernatant \\ QBtE264 supernatant}

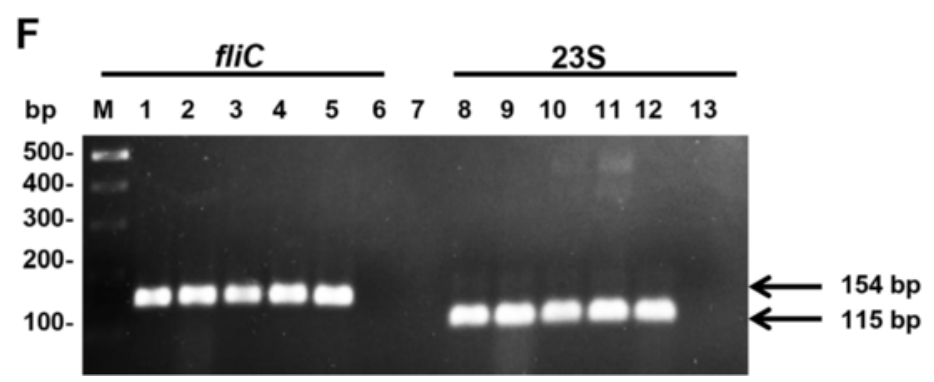

Figure 5 Live-cell imaging analysis to track the movement of B. thailandensis E264. (A) B. thailandensis E264 (BtE264); BtE264 exposed to cell-free supernatants from: (B), B. pseudomallei 1710a; (C), B. pseudomallei K96243 and (D), B. thailandensis E264. Twenty cells were randomly tracked for movement by light microscopy and their paths of movement were determined from video recording using an ImageJ program (http://rsb.info.nih.gov/ij/). Colored lines show the paths of different cells over a $20 \mathrm{sec}$ period. (E) Motility distance of B. thailandensis untreated BtE264 (non-exposed control) and after exposure to cell-free supernatants from B. pseudomallei 1710a, B. pseudomallei K96243 and B. thailandensis E264 control. (F) Expression of fliC RNA and 23S rRNA of B. thailandensis E264 exposed to cell-free supernatant of B. pseudomallei. RT-PCR of fliC RNA (lane 1-4) and 23S rRNA (lane 8-11) of B. thailandensis E264 colony exposed to cell-free supernatant of B. pseudomallei 1710a (lane 1 and 8), B. pseudomallei K96243 (lane 2 and 9), B. thailandensis E264 (lane 3 and 10), and non-exposed control (lane 4 and 11). Lanes 5 and 12 are PCR of genomic DNA controls for fliC and 23S rRNA primers respectively. Lanes 6 and 13 are no RT negative controls. 


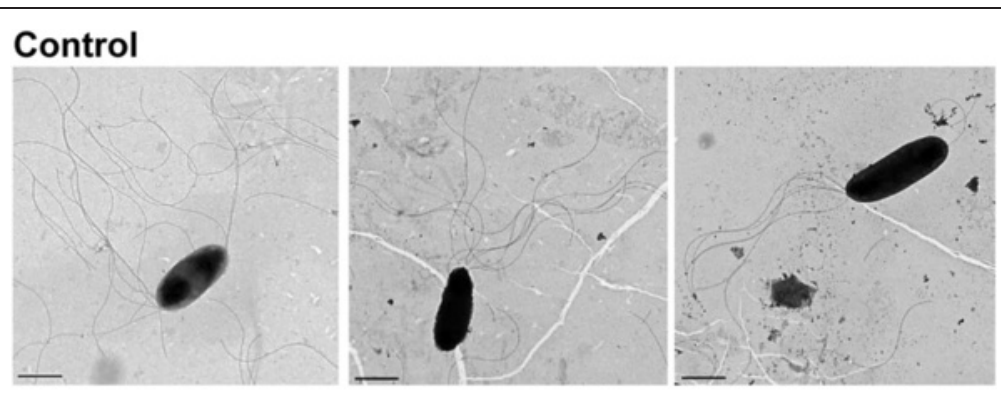

\section{Bp1710a supernatant}

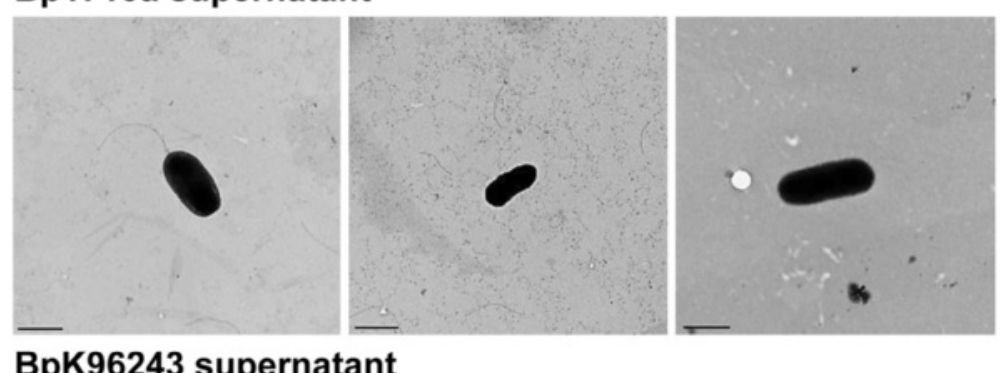

\section{BpK96243 supernatant}
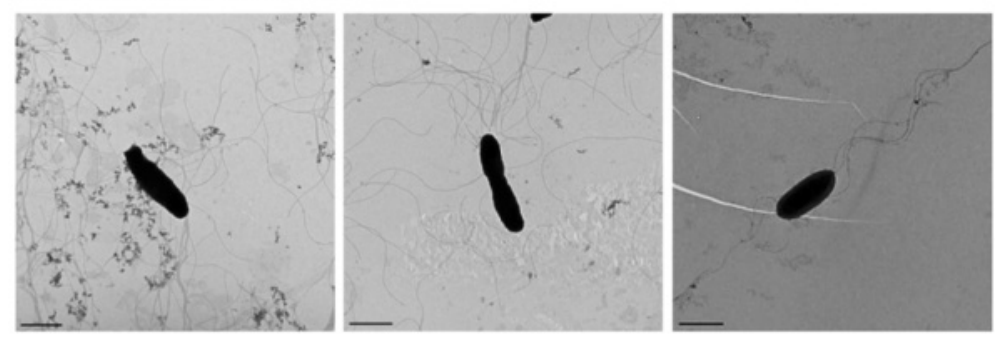

\section{BtE264 supernatant}
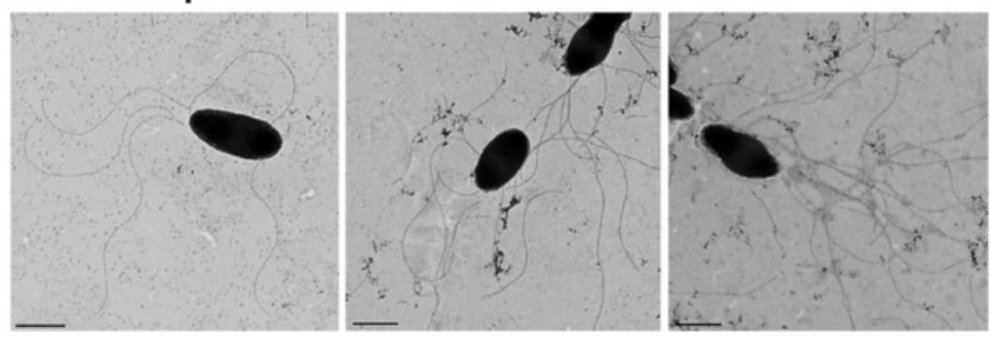

Figure 6 Transmission electron microscopy (TEM) of $B$. thailandensis E264 unexposed control, or exposed to cell-free supernatant of $B$. pseudomallei 1710 a, B. pseudomallei K96243 or B. thailandensis E264, respectively. Bacterial cells were negatively stained with $1 \%$ uranyl

acetate and visualized by TEM. Scale bars illustrate $1 \mu \mathrm{m}$.

thailandensis [20]. In addition, there are 30 differences in amino acid distributed across the flagellin sequence. A recent report of the comparison between flagellin proteins of these two species also showed that their flagellins was modified with different masses of glycan (291 Da for B. pseudomallei and 300 or $342 \mathrm{Da}$ for B. thailandensis) [21]. It is possible that these factors may be implicated in the different susceptibility to the inhibition activity of $B$. pseudomallei.

The ability of $B$. pseudomallei to survive in diverse environments is likely to be associated with a genetic repertoire that facilitates competition and adaptation [22]. This includes the presence of at least 10 CDI types that have been identified in the genomes of sequenced $B$. pseudomallei strains [10]. Our results are consistent with the hypothesis that the growth inhibition we observed on LB agar was caused by one or more CDI or T6SS systems, since B. pseudomallei culture supernatants had no effect on growth of $B$. thailandensis. Previous work demonstrated that B. pseudomallei CdiA-CT toxins are functional [10]. For example, the CdiA-CT of B. pseudomallei exhibits tRNase activity, which blocks the growth of $B$. thailandensis E264 target cells [10]. It is also possible that another mechanism of contact-dependent growth inhibition may be involved such as Type VI secretion systems (T6SS) that are present in B. pseudomallei [9]. 
Our analysis suggests that this growth inhibition phenomenon was not the result of different growth rates of B. pseudomallei and B. thailandensis. Inhibition of $B$. thailandensis by $B$. pseudomallei 1710a was only observed at a ratio greater than 1:1, suggesting that the B. pseudomallei inhibitor may mediate growth inhibition in a cell-density dependent or in a co-operative manner. Such cooperation has not been reported for $B$. pseudomallei, but it has been reported that the CDI genes in B. thailandensis were expressed in a subpopulation during culture in broth and also played a role in biofilm formation $[11,23]$.

\section{Conclusion}

Our results indicate that B. pseudomallei inhibits both the growth and motility of its close relative $B$. thailandensis. The latter phenomenon appears to occur via a previously unreported mechanism involving an effect on flagellar structure.

\section{Methods \\ Bacterial isolates}

Sixty-seven B. pseudomallei and six B. thailandensis isolates were examined in this study. The B. pseudomalle $i$ isolates originated from Thailand (thirty-six from human infection and twenty-seven from the environment) or Australia (three from human infection, and one from the environmental water) $[4,24]$ (Additional file 1: Table S1). Five B. thailandensis (E29, E175, E264, E421 and E426) were isolated from soil in central Thailand and the sixth isolate was B. thailandensis Bt6, a kanamycin resistant mutant derived previously from B. thailandensis E264 (E264 attTN7::miniTn7T-Kan) [10]. A further fifteen $B$. pseudomallei strains were tested, which were derived from five isolates $(153,164$ \& K96243 from human disease in Thailand, B3 and B4 from the environment in Thailand) [4,12] (Additional file 1: Table S1). This included wild type (type I), together with two isogenic colony morphology variants (types II \& III) for each isolate, which were generated from type I of each strain using nutritional limitation $[12,13]$. Bacteria were grown in Luria-Bertani (LB) broth or on LB agar and incubated at $37^{\circ} \mathrm{C}$ in air. $\mathrm{LB}$ with $500 \mu \mathrm{g} / \mathrm{ml}$ kanamycin (Kan) was used to culture $B$. thailandensis Bt6. The study was granted exemption from requiring ethics approval by Ethics Committee of Faculty of Tropical Medicine, Mahidol University.

\section{Growth curves}

A single bacterial colony was suspended in sterile phosphate buffered saline (PBS) and adjusted to an $\mathrm{OD}_{600}$ of 0.15 to obtain $1 \times 10^{8} \mathrm{cfu} / \mathrm{ml}$. One hundred microlitres of bacterial suspension was added to $10 \mathrm{ml}$ of LB broth and incubated at $37^{\circ} \mathrm{C}$ in air with shaking at $200 \mathrm{rpm}$ for
$24 \mathrm{~h}$. At $2 \mathrm{~h}$ intervals, $100 \mu \mathrm{l}$ of bacterial culture was collected, serially diluted 10-fold in PBS, and the number of viable cells counted by plating on LB agar or LB agar containing $500 \mu \mathrm{g} / \mathrm{ml} \mathrm{Kan} \mathrm{(for} \mathrm{B.} \mathrm{thailandensis} \mathrm{Bt6)} \mathrm{in}$ triplicate. Plates were incubated at $37^{\circ} \mathrm{C}$ in air for 2 days and doubling time was calculated.

\section{Growth inhibition determined by broth microdilution assay}

A broth microdilution assay was used to determine the effect of cell-free supernatant from B. pseudomallei on growth of B. thailandensis. B. pseudomallei supernatant from overnight culture in $\mathrm{LB}$ broth at $37^{\circ} \mathrm{C}$ was concentrated using a filter membrane with 10,000 cut-off (Millipore, Billerica, MA, USA) to $5 \times, 2.5 \times$ and $1.2 \times$, and an equal volume of each concentrate added to $50 \mu \mathrm{l}$ of an overnight culture of B. thailandensis to obtain a final concentration $5 \times 10^{5} \mathrm{cfu} / \mathrm{ml}$ in LB. The control was performed by adding an equal volume of PBS to $50 \mu \mathrm{l}$ of an overnight culture of B. thailandensis. The experiment was performed in a 96-well plate in triplicate in a given experiment. Cultures were incubated at $37^{\circ} \mathrm{C}$ in air for $18 \mathrm{~h}$. Bacterial growth was defined as visual turbidity followed by colony count.

\section{Swarming motility inhibition assay}

Three methods were devised to test whether interactions between $B$. pseudomallei and B. thailandensis affected swarming motility. (i) A colony of B. pseudomallei and a colony of $B$. thailandensis obtained from an overnight culture on LB agar were spotted using a pipette tip at opposite poles of a swarm agar plate, and the growth observed daily over a 3 day incubation time course at $37^{\circ} \mathrm{C}$. (ii) Supernatant from an 18 hour B. pseudomallei culture in LB broth was harvesting using centrifugation and filtrated through $0.2 \mu \mathrm{m}$ membrane (Sartorius, Goettingen, Germany). A colony of $B$. thailandensis from overnight culture on LB agar was spotted onto the center of the plate and incubated at $37^{\circ} \mathrm{C}$ for $16 \mathrm{~h}$, after which $100 \mu \mathrm{l}$ of cell-free B. pseudomallei supernatant was dropped onto the plate at 2, 6 and 10 o'clock and 1-1.5 cm from the edge of the plate. The plate was further incubated for $16 \mathrm{~h}$, and then observed for inhibition of B. thailandensis swarming, which was defined as the presence of any clear zone around the supernatant drop. We spotted the supernatant of B. pseudomallei after B. thailandensis was allowed to swarm for a period of time because the inhibition activity was lost if applied at the beginning of the assay. (iii) $100 \mu \mathrm{l}$ of cell-free B. pseudomallei supernatant was dropped in the center of a swarm plate and left to diffuse and dry in air for $15 \mathrm{~min}$. The same spot was then inoculated with a $B$. thailandensis colony using a pipette tip, and the plate then incubated for $18 \mathrm{~h}$ before the swarm $B$. thailandensis colony was examined. 


\section{Growth inhibition assay on agar plates}

A growth inhibition assay was carried out as described previously [10]. In brief, bacteria were grown for $4 \mathrm{~h}$ to early log phase (OD $600 \mathrm{~nm}=0.2-0.5$ ) in LB or LB with Kan. Bacterial concentration was adjusted using OD, $B$. pseudomallei mixed with $B$. thailandensis in a ratio of $1000: 1,100: 1,10: 1$ or $1: 1$, and $100 \mu \mathrm{l}$ of the mixture dropped onto a $2.5 \times 2.5 \mathrm{~cm}^{2}$ sterile nitrocellulose membrane which was placed on top of LB agar. Plates were incubated at $37^{\circ} \mathrm{C}$ in air for $24 \mathrm{~h}$ after which the nitrocellulose membrane was removed, placed into $10 \mathrm{ml}$ PBS and vortexed vigorously for $30 \mathrm{sec}$ to harvest bacteria. Duplicate plating onto LB and LB plus Kan was performed, and plate counts determined for B. thailandensis Bt6 (LB/Kan plate) and B. pseudomallei (total count on LB minus B. thailandensis count on LB/Kan). To examine the effect of incubation time, a co-culture of B. pseudomallei 1710a and B. thailandensis Bt6 at a ratio of 1000:1 was incubated at $37^{\circ} \mathrm{C}$ for $3 \mathrm{~h}, 6 \mathrm{~h}$ and $24 \mathrm{~h}$. At the indicated time point, cells were harvested from the plates and viable bacteria counted as before. The interaction was also tested between $B$. thailandensis Bt6 and six B. pseudomallei isolates (K96243, H1244a, 1106b, 1026b, 1710a, B4), in which B. thailandensis E264 wild type was used for comparison using a ratio of B. thailandensis E264 to B. thailandensis Bt6 at 1000:1 for $24 \mathrm{~h}$. Three B. pseudomallei isolates possessed different CDI types (strains K96243, type I; 1710a, type II; and 1026b, type V and VIII) [10]. All assays were performed in triplicate in a given experiment.

\section{Separation and preparation of concentrated motility inhibitory factor}

B. pseudomallei was inoculated into LB and incubated overnight at $37^{\circ} \mathrm{C}$ in air with shaking at $200 \mathrm{rpm}$. The bacterial culture was centrifuged at $4,000 \times \mathrm{g}$ for $15 \mathrm{~min}$ at $4^{\circ} \mathrm{C}$ and the supernatant filtrated through a $0.2 \mu \mathrm{m}$ membrane. To estimate the molecular size of the inhibitory substance, $10 \mathrm{ml}$ of the cell-free supernatant of $B$. pseudomallei 1710a was filtrated through filter membranes with a molecular weight cut-off (MWCO) of $10,000,30,000,50,000$ or 100,000 daltons (Millipore, Billerica, MA, USA) to obtain 15-100 times concentrated culture supernatant. The presence of inhibitory substance in the retentate or filtrate after membrane separation was assessed using the inhibition motility assay (method (ii)).

\section{Protein digestion of inhibitory molecule}

The protein nature of the inhibitory factor was characterized by digestion with two different proteases. Cellfree supernatant of $18 \mathrm{~h}$ culture at $37^{\circ} \mathrm{C}$ in $\mathrm{LB}$ broth of $B$. pseudomallei 1710a was concentrated 15 times using 30,000 membrane filtration, quantified using the bicinchoninic acid assay (Pierce, Rockford, IL, USA), and analyzed by SDS-PAGE [25]. The sample was diluted to $1 \mathrm{mg} / \mathrm{ml}$ and digested at an enzyme: substrate ratio of $1: 10(\mathrm{w} / \mathrm{w})$ with proteinase $\mathrm{K}$ (Invitrogen, Carlsbad, CA, USA) or pronase (Merck, Nottingham, UK) in the relevant buffer as recommended by the manufacturer (proteinase $\mathrm{K}$ in PBS at $37^{\circ} \mathrm{C}$ for $72 \mathrm{~h}$ and pronase in PBS containing $10 \mathrm{mM} \mathrm{CaCl} 2$ at $37^{\circ} \mathrm{C}$ for $72 \mathrm{~h}$ ).

\section{Assays for stability of inhibitory factor}

Inhibition activity of cell-free supernatant of B. pseudomallei 1710a was tested after adjustment to a $\mathrm{pH}$ of 3,4 , $5,6,7$ or 8 , and after being maintaining at $\mathrm{pH} 7.0$ at $80^{\circ} \mathrm{C}, 4^{\circ} \mathrm{C}, 25^{\circ} \mathrm{C}, 37^{\circ} \mathrm{C}, 50^{\circ} \mathrm{C}$ or $80^{\circ} \mathrm{C}$ for $24 \mathrm{~h}$. Inhibition activity was also tested at $\mathrm{pH} 7.0$ in the presence of $\mathrm{NaCl}$ at a final concentration of $0.2 \mathrm{M}, 0.4 \mathrm{M}, 0.6 \mathrm{M}, 0.8 \mathrm{M}$ or $1 \mathrm{M}$. Aliquots of $100 \mu \mathrm{l}$ were tested in the B. thailandensis E264 inhibition motility assay at $37^{\circ} \mathrm{C}$ for $18 \mathrm{~h}$ (method ii). Untreated overnight cell-free supernatant of B. pseudomallei 1710a in LB broth was used as a positive control and LB broth was used as a negative control. Results for inhibition of $B$. thailandensis motility were read based on the presence (positive) or absence (negative) of a clear zone on swarm agar.

\section{Visualization of $B$. thailandensis motility and flagella}

Live cell imaging was used to examine the motility of $B$. thailandensis E264 after exposure to cell-free supernatant from B. pseudomallei 1710a, B. pseudomallei K96243 and B. thailandensis E264 (control). The method was modified from a previous study [26]. B. thailandensis cells were exposed to B. pseudomallei cell-free supernatant using the swarming inhibition motility method (iii). B. thailandensis E264 was picked from the colony at the end of this assay and suspended in $50 \mu \mathrm{l}$ PBS. Two microlitres of bacterial suspension was overlaid on a film of $1.5 \%$ low-melting agarose pad (Invitrogen, Carlsbad, CA, USA) on a microscope glass slide. A cover slip was placed and motility was observed by light microscope (Leica DM750, Wetzlar, Germany) with $100 \times$ magnification. Video was recorded and 20 individual cells were tracked and analyzed for $20 \mathrm{sec}$ motility using ImageJ program (http://rsb.info.nih.gov/ij/) with the manual tracking plug-in.

The same B. thailandensis E264 cell preparation was examined using TEM for the presence of flagella. Fifty microliters of the $B$. thailandensis suspension was dropped onto parafilm and Fomvar coated carbon grids were placed on top for $10 \mathrm{~min}$ to transfer bacterial cells. The liquid was carefully removed with filter paper and the samples were stained with $1 \%$ uranyl acetate for 10 min after which the liquid was again carefully removed. The grid was dried at room temperature for 
overnight. Bacteria were observed with a Hitachi Electron Microscope H-7000 (Japan). The presence of bacterial flagella was recorded for 100 bacteria per isolate.

\section{Reverse transcriptase PCR for fliC gene expression}

B. thailandensis E264 cells were picked from a colony at the completion of method (iii) as above. RNA was extracted using Trizol reagent, as described previously [27]. Primers were designed for $f l i C$ of $B$. thailandensis E264 (accession number AF081500.1) using PrimerBLAST (http://www.ncbi.nlm.nih.gov/tools/primer-blast). One-step reverse transcriptase (RT)-PCR was performed using $1 \mu \mathrm{g}$ RNA and a Superscript III One-step RT-PCR system (Invitrogen, Carlsbad, CA, USA) with forward primer 5' GGTCGCTCAACAGAACCTCA 3' and reverse primer 5' CTGGTTCAGGCCGTTGATCT3'. The RTPCR conditions were as follows: cDNA synthesis at $45^{\circ} \mathrm{C}$ for $30 \mathrm{~min}$; initial denaturation at $95^{\circ} \mathrm{C}$ for $5 \mathrm{~min}$; 30 cycles of denaturation at $94^{\circ} \mathrm{C}$ for $30 \mathrm{sec}$, annealing at $50^{\circ} \mathrm{C}$ for $15 \mathrm{sec}$, and extension at $72^{\circ} \mathrm{C}$ for $30 \mathrm{sec}$; and a final elongation step at $72^{\circ} \mathrm{C}$ for $7 \mathrm{~min}$. The positive control was RT-PCR for 23S rRNA amplification using primers 23S_F and 23S_R with forward primer 5' GTAGACCC GAAACCAGGTGA3' and reverse primer 5' CACCCC TATCCACAGCTCAT 3'. The negative control was a reaction without $\mathrm{RT}$ enzyme. The amplified product was run on a $1.5 \%$ agarose gel, stained with ethidium bromide and visualized under UV light.

\section{Statistical analysis}

Statistical analyses were performed using Stata, version 12 (StataCorp LP, College Station, TX, USA). Fisher's exact test or Chi square tests were used to test proportions. One-way ANOVA was used to test the difference between groups. Quantitative data are presented as mean \pm standard deviation. Differences were considered statistically significant at a p-value $\leq 0.05$.

\section{Additional file}

Additional file 1: Table S1. Summary of B. pseudomallei isolates and inhibitory activity against $B$. thailandensis swarming motility.

\section{Competing interests}

The authors declare that they have no competing interests.

\section{Authors' contributions}

WW collected and provided B. pseudomallei and B. thailandensis strains. DL provided Bt6 isolate and technical advice. WN, ST, CW, JS and OR performed experiments. NC and WN designed experiment and performed data analysis. NC, DL and SJP formulated the hypothesis and wrote the paper. All authors reviewed and approved the final draft.

\section{Acknowledgments}

We are grateful for support from staff at Sappasithiprasong Hospital and the Mahidol-Oxford Tropical Medicine Research Unit. We thank Urai Chaisri and Supatra Suwanmanee (Department of Tropical Pathology) for their assistance with TEM, Khemaporn Boonbumrung (Chulalongkorn University), David W. Rice, Svetlana E. Sedelnikova (University of Sheffield), Herbert Schweizer (Cololado State University) and Paul Williams (University of Nottingham) for technical advice, and Premjit Amornchai for strain subculture. We thank Bart Currie for providing B. pseudomallei isolates from Australia. The authors acknowledge Direk Limmathurotsakul, Muthita Vanaporn, Pornpan Pumirat and Janjira Thaipadungpanit for their comments.

This study was funded by a Wellcome Trust Career Development award in Public Health and Tropical Medicine, UK (grant 087769/Z/08/Z to NC). The funder had no role in study design, data collection and analysis, decision to publish, or preparation of the manuscript.

\section{Author details}

'Department of Microbiology and Immunology, Faculty of Tropical Medicine, Mahidol University, 420/6 Rajvithi Road, Bangkok 10400, Thailand. ${ }^{2}$ Mahidol-Oxford Tropical Medicine Research Unit, Faculty of Tropical Medicine, Mahidol University, Bangkok, Thailand. ${ }^{3}$ Department of Molecular Tropical Medicine and Genetics, Faculty of Tropical Medicine, Mahidol University, Bangkok, Thailand. ${ }^{4}$ Department of Molecular, Cellular, and Developmental Biology, University of California, Santa Barbara, CA, USA. ${ }^{5}$ Biomolecular Science and Engineering Program, University of California, Santa Barbara, CA, USA. ${ }^{6}$ Department of Medicine, University of Cambridge, Addenbrooke's Hospital, Cambridge, UK.

Received: 8 December 2014 Accepted: 19 February 2015

Published online: 03 March 2015

\section{References}

1. Limmathurotsakul D, Dance DA, Wuthiekanun V, Kaestli M, Mayo M, Warner $J$, et al. Systematic review and consensus guidelines for environmental sampling of Burkholderia pseudomallei. PLoS Negl Trop Dis. 2013;7(3):e2105. doi: 10.1371/journal.pntd.0002105.

2. Limmathurotsakul D, Kanoksil M, Wuthiekanun V, Kitphati R, DeStavola B, Day NP, et al. Activities of daily living associated with acquisition of melioidosis in northeast Thailand: a matched case-control study. PLoS Negl Trop Dis. 2013;7(2):e2072. doi:10.1371/journal.pntd.0002072.

3. Wiersinga WJ, Currie BJ, Peacock SJ. Melioidosis. N Engl J Med. 2012; 367(11):1035-44. doi: 10.1056/NEJMra1204699.

4. Chantratita N, Wuthiekanun V, Limmathurotsakul D, Vesaratchavest M, Thanwisai A, Amornchai P, et al. Genetic diversity and microevolution of Burkholderia pseudomallei in the environment. PLoS Negl Trop Dis. 2008;2(2):e182. doi: 10.1371/journal.pntd.0000182.

5. Brett PJ, Deshazer D, Woods DE. Characterization of Burkholderia pseudomallei and Burkholderia pseudomallei-like strains. Epidemiol Infect. 1997;118(2):137-48.

6. Smith MD, Angus BJ, Wuthiekanun V, White NJ. Arabinose assimilation defines a nonvirulent biotype of Burkholderia pseudomallei. Infect Immun. 1997;65(10):4319-21.

7. Trakulsomboon S, Vuddhakul V, Tharavichitkul P, Na-Gnam N, Suputtamongkol $Y$, Thamlikitkul V. Epidemiology of arabinose assimilation in Burkholderia pseudomallei isolated from patients and soil in Thailand. Southeast Asian J Trop Med Public Health. 1999;30(4):756-9.

8. Duerkop BA, Varga J, Chandler JR, Peterson SB, Herman JP, Churchill ME, et al. Quorum-sensing control of antibiotic synthesis in Burkholderia thailandensis. J Bacteriol. 2009;191(12):3909-18. doi: 10.1128/JB.00200-09.

9. Schwarz S, West TE, Boyer F, Chiang WC, Carl MA, Hood RD, et al. Burkholderia type VI secretion systems have distinct roles in eukaryotic and bacterial cell interactions. PLoS Pathog. 2010;6(8):e1001068. doi: 10.1371/ journal.ppat.1001068.

10. Nikolakakis K, Amber S, Wilbur JS, Diner EJ, Aoki SK, Poole SJ, et al. The toxin/immunity network of Burkholderia pseudomallei contact-dependent growth inhibition (CDI) systems. Mol Microbiol. 2012;84(3):516-29. doi: 10.1111/j.1365-2958.2012.08039.x.

11. Anderson MS, Garcia EC, Cotter PA. The Burkholderia bcpAIOB genes define unique classes of two-partner secretion and contact dependent growth inhibition systems. PLoS Genet. 2012;8(8):e1002877. doi: 10.1371/journal. pgen. 1002877

12. Chantratita N, Wuthiekanun V, Boonbumrung $K$, Tiyawisutsri $R$, Vesaratchavest M, Limmathurotsakul D, et al. Biological relevance of colony morphology and phenotypic switching by Burkholderia pseudomallei. J Bacteriol. 2007: 189(3):807-17. doi: JB.01258-06. 
13. Tandhavanant S, Thanwisai A, Limmathurotsakul D, Korbsrisate S, Day NP, Peacock SJ, et al. Effect of colony morphology variation of Burkholderia pseudomallei on intracellular survival and resistance to antimicrobial environments in human macrophages in vitro. BMC Microbiol. 2010;10:303. doi: 10.1186/1471-2180-10-303.

14. Manivanh L, Pierret A, Rattanavong S, Buisson Y, Elliott I, Maeght J-L, et al. Burkholderia pseudomallei in a rice paddy in Northern Laos-influence of depth, season and physicochemical parameters. Bangkok, Thailand: 7th World Melioidosis Congress 2013; 2013. p. 193.

15. Kaestli M, Schmid M, Mayo M, Rothballer M, Harrington G, Richardson L, et al. Out of the ground: aerial and exotic habitats of the melioidosis bacterium Burkholderia pseudomallei in grasses in Australia. Environ Microbiol. 2012;14(8):2058-70. doi: 10.1111/j.1462-2920.2011.02671.x.

16. Boonbumrung K, Wuthiekanun V, Rengpipat S, Day NP, Peacock SJ. In vitro motility of a population of clinical Burkholderia pseudomallei isolates. J Med Assoc Thai. 2006;89(9):1506-10.

17. DeShazer D, Brett PJ, Carlyon R, Woods DE. Mutagenesis of Burkholderia pseudomallei with Tn5-OT182: isolation of motility mutants and molecular characterization of the flagellin structural gene. J Bacteriol. 1997;179(7):2116-25.

18. Vesaratchavest M, Tumapa S, Day NP, Wuthiekanun V, Chierakul W, Holden MT, et al. Nonrandom distribution of Burkholderia pseudomallei clones in relation to geographical location and virulence. J Clin Microbiol. 2006:44(7):2553-7. doi: 44/7/2553.

19. Winstanley C, Morgan JA. The bacterial flagellin gene as a biomarker for detection, population genetics and epidemiological analysis. Microbiology. 1997;143(Pt 10):3071-84.

20. Wajanarogana S, Sonthayanon P, Wuthiekanun V, Panyim S, Simpson AJ, Tungpradabkul S. Stable marker on flagellin gene sequences related to arabinose non-assimilating pathogenic Burkholderia pseudomallei. Microbiol Immunol. 1999:43(11):995-1001.

21. Scott AE, Twine SM, Fulton KM, Titball RW, Essex-Lopresti AE, Atkins TP, et al. Flagellar glycosylation in Burkholderia pseudomallei and Burkholderia thailandensis. J Bacteriol. 2011;193(14):3577-87. doi: 10.1128/JB.01385-10.

22. Holden MT, Titball RW, Peacock SJ, Cerdeno-Tarraga AM, Atkins T, Crossman $L C$, et al. Genomic plasticity of the causative agent of melioidosis, Burkholderia pseudomallei. Proc Natl Acad Sci U S A. 2004;101(39):14240-5. doi: 10.1073/ pnas.0403302101.

23. Garcia EC, Anderson MS, Hagar JA, Cotter PA. Burkholderia BcpA mediates biofilm formation independently of interbacterial contact-dependent growth inhibition. Mol Microbiol. 2013;89(6):1213-25. doi: 10.1111/mmi.12339.

24. Wuthiekanun V, Limmathurotsakul D, Chantratita N, Feil EJ, Day NP, Peacock SJ. Burkholderia pseudomallei is genetically diverse in agricultural land in Northeast Thailand. PLoS Negl Trop Dis. 2009;3(8):e496. doi: 10.1371/journal. pntd.0000496.

25. Laemmli UK. Cleavage of structural proteins during the assembly of the head of bacteriophage T4. Nature. 1970;227(5259):680-5.

26. De Jong IG, Beilharz K, Kuipers OP, Veening JW. Live cell imaging of Bacillus subtilis and Streptococcus pneumoniae using automated time-lapse microscopy. J Vis Exp. 2011(53). doi: 10.3791/3145.

27. Chantratita N, Tandhavanant S, Wikraiphat C, Trunck LA, Rholl DA, Thanwisai $A$, et al. Proteomic analysis of colony morphology variants of Burkholderia pseudomallei defines a role for the arginine deiminase system in bacterial survival. J Proteomics. 2012;75(3):1031-42. doi: 10.1016/j.jprot.2011.10.015.

\section{Submit your next manuscript to BioMed Central and take full advantage of:}

- Convenient online submission

- Thorough peer review

- No space constraints or color figure charges

- Immediate publication on acceptance

- Inclusion in PubMed, CAS, Scopus and Google Scholar

- Research which is freely available for redistribution

Submit your manuscript at www.biomedcentral.com/submit 